\title{
Efeito dose-resposta de fatores de risco para a doença isquêmica do coração*
}

\section{Dose-response effect of risk factors to ischaemic heart disease}

\author{
Suzana Alves de Moraes e José Maria Pacheco do Souza \\ Departamento de Epidemiologia da Faculdade de Saúde Pública da Universidade de São Paulo. São \\ Paulo, SP-Brasil.
}

\begin{abstract}
Resumo
Resultados de diversos estudos têm apontado a relevância da hipertensão arterial, do hábito de fumar e da hipercolesterolemia como fatores de risco para a doença isquêmica do coração (DIC). Poucos autores têm investigado a existência de gradiente linear relacionando a quantidade destas exposições com os eventos coronarianos. Com o objetivo de avaliar o efeito de diferentes graus de exposição a estas variáveis sobre a DIC, procedendo-se ao ajustamento para possíveis variáveis de confusão, foi feito estudo planejado sob a forma de desenho tipo casocontrole, tendo a coleta de dados se estendido de março de 1993 a fevereiro de 1994. Foram estudados 833 indivíduos de ambos os sexos, na faixa etária compreendida entre 30 e 69 anos completos, sendo todos residentes no Município de São Paulo, SP (Brasil). Foram comparados 280 casos com 553 controles (285 controles de vizinhança e 268 controles hospitalares). A técnica estatística utilizada para a análise dos dados foi a regressão logística multivariada. Os resultados permitiram identificar gradiente linear para as variáveis duração da hipertensão arterial e para número de cigarros consumidos/dia. As variáveis duração do hábito de fumar e duração da hipercolesterolemia, embora tendo apresentado "odds ratios" significantes para as respectivas categorias de exposição, não apresentaram gradiente linear. Foram discutidos aspectos metodológicos que poderiam exercer influência sobre a tendência dos "odds ratios" nas categorias de exposição das variáveis duração do hábito de fumar e duração da hipercolesterolemia. Conclui-se que os efeitos dose-resposta observados para as variáveis duração da hipertensão arterial e número de cigarros consumidos/dia foram independentes da presença nos modelos de potentes fatores de risco para a doença isquêmica do coração.
\end{abstract}

Isquemia miocárdica. Estudos de casos e controles. Fatores de risco.

\begin{abstract}
Several authors have reported hypertension, smoking and hypercholesterolemia as independent risk factors to ischaemic heart disease (IHD). However few of them have investigated the existence of a linear gradient related to the levels of these exposures and IHD. The effect of different levels of these exposures and IHD after adjusting for known confounders of effect, is assessed. The
\end{abstract}

\footnotetext{
* Parte da tese de doutorado: "Diabetes mellitus e doença isquêmica do coração: um estudo tipo caso-controle", apresentada ao Departamento de Epidemiologia da Faculdade de Saúde Pública da Universidade de São Paulo, 1995. Projeto financiado pela Fundação de Amparo à Pesquisa do Estado de São Paulo. Processo nº $92.2558-0$.

Correspondência para/Correspondence to: Suzana Alves de Moraes - Rua Albert Einstein, 1334/12 - 14051-110 Ribeirão Preto, SP - Brasil. E-mail: samoraes@usp.br

Edição subvencionada pela FAPESP. Processo 95/2290-6

Recebido em 25.9.1995. Aprovado em 28.2.1996.
} 
project was designed as a case-control study and the data were collected over one year from March/93 to February/94. The sample was composed of a total of 833 individuals of both genders aged 30-69 living in the city of S. Paulo, SP (Brazil), 280 of whom were compared with 553 controls (285 neighbourhood controls and 268 hospital controls). Logistic regression was the statistical method wold for the analysis of the data. The results showed a linear gradient for known duration of hypertension and daily number of cigarettes consumed. Although the variables duration of hypercholesterolemia and duration of the habit smoking presented statistically significant odds ratio in the respective strata there was no indication of a linear gradient. Some methodological issues are presented to explain this absence of a linear gradient for known duration of hypercholesterolemia and duration of the smoking habit. It is concluded that the dose response effect detected for known duration of hypertension and daily number of cigarettes consumed were independent of the presence of major risk factors ischaemic heart disease.

\section{Myocardial ischemia. Case-control studies. Risk factors.}

\section{INTRODUÇÃO}

O atual estágio da transição demográfico-epidemiológica pelo qual atravessam as sociedades em desenvolvimento, ao lado da adoção de um moderno estilo de vida, tem-lhes conferido mudança nos padrões de morbi-mortalidade em direção às doenças crônico-degenerativas, entre as quais se destacam o diabetes mellitus, a hipertensão arterial, a doença isquêmica do coração, a obesidade e a gota ${ }^{18,20}$.

Lolio col. ${ }^{9}$, estudando a mortalidade por doença isquêmica do coração (DIC), no Município de São Paulo, no período de 1970 a 1983, observaram queda significante nos coeficientes ajustados por idade no período, embora a comparação com as respectivas taxas de 27 países industrializados mantivesse o Município de São Paulo próximo àqueles países com as maiores taxas de mortalidade por essa causa.

Entre as inúmeras condições associadas à DIC, apontadas na literatura, parece haver unanimidade em considerar a hipertensão arterial, a hipercolesterolemia e o hábito de fumar como os mais potentes fatores de risco para a doença isquêmica do coração ${ }^{4,7,21}$. A investigação, todavia, de um efeito dose-resposta, relacionando diferentes níveis de exposição a estes fatores de risco com a DIC, não tem sido constante, subtraindo-se, desta forma, a possibilidade de se identificar o efeito de gradação. $\mathrm{O}$ efeito dose-resposta para a duração da hipertensão arterial foi apontado por alguns autores ${ }^{5,13,15,16,19}$, não se tendo encontrado, em publicações dos últimos 10 anos, trabalhos que relacionassem o efeito da duração da hipercolesterolemia com a doença isquêmica do coração.

Dentro desta perspectiva, o estudo teve por objetivo avaliar a existência de um efeito dose-resposta entre diferentes níveis de exposição a estes fatores de risco e a doença isquêmica do coração, procedendo-se ao ajustamento simultâneo para possíves variáveis de confusão.

\section{MATERIAL E MÉTODO}

O estudo foi planejado sob a forma de um desenho tipo caso-controle ${ }^{17}$ e a coleta de dados estendeu-se de março de 1993 a fevereiro de 1994. Foram estudados 833 indivíduos de ambos os sexos, na faixa etária compreendida entre 30 e 69 anos completos, sendo todos residentes no Município de São Paulo. Foram comparados 280 casos com 553 controles (285 controles de vizinhança e 268 controles hospitalares). Os dados foram retirados de um estudo caso-controle ${ }^{11,12}$ cuja proposta original envolveu uma investigação epidemiológica mais ampla, bem como a análise de alguns aspectos metodológicos relativos a este tipo de delineamento, não sendo, em seu todo, objeto do presente estudo.

\section{Definição e Seleção de Casos}

Foram considerados casos os indivíduos egressos do Pronto Socorro de um hospital especializado em doenças do coração, entre os meses de março e maio de 1993, em cujas fichas médicas de alta hospitalar constassem, como diagnóstico primário ou secundário, os códigos compreendidos entre 410 e 414 da Classificação Internacional de Doenças, IX Revisão ${ }^{14}$. O acesso a estas fichas se deu a partir do serviço de arquivo médico e estatística hospitalar do hospital estudado, que forneceu as fichas de alta hospitalar de cada paciente, a partir das quais um médico treinado procedeu à seleção. Os casos elegíveis para o estudo foram selecionados por ordem cronológica de alta hospitalar, até que se completasse o número previsto para o tamanho da amostra. Procedeu-se inicialmente ao cadastramento dos casos e mapeamento dos respectivos locais de residência, onde foi realizada uma entrevista 
padronizada. Em situações que impediram a entrevista do caso selecionado, como óbito, não-localização do endereço ou recusa em participar do estudo, foi feita a substituição, priorizando-se, para a entrevista, um caso com data de alta hospitalar próxima àquela do caso excluído.

\section{Definição e Seleção de Controles}

A cada semana foi confeccionada uma listagem contendo a distribuição por sexo e faixa etária dos casos entrevistados. Cada caso foi emparelhado por sexo e faixa etária (intervalo de 5 anos) com dois tipos de controles: um controle de vizinhança e um controle hospitalar, tendo sido previamente estabelecido que, para fazer parte do grupo-controle, os indivíduos não poderiam apresentar história atual ou pregressa de doença isquêmica do coração e/ou outras cardiopatias. Os controles de vizinhança foram selecionados a partir dos subdistritos de residência dos respectivos casos. Não foram selecionados controles da mesma residência do caso ou que apresentassem, em relação a este, algum grau de parentesco. Os controles hospitalares foram selecionados do ambulatório geral e didático do complexo hospitalar ao qual se vincula o hospital estudado, cuja demanda se caracteriza pelo atendimento de pacientes de clínica geral em regime de pronto atendimento.

\section{Coleta dos Dados}

A detecção das exposições foi procedida mediante entrevistas, utilizando-se um questionário pré-codificado, aplicado e preenchido por uma equipe de entrevistadores previamente treinada. Os entrevistadores eram pessoas com formação de nível superior ou estudantes universitários cursando, pelo menos, o terceiro ano de graduação. A nenhum dos entrevistadores foram dadas a conhecer as hipóteses do estudo. A duração média de cada entrevista foi de $40 \mathrm{~min}$. As entrevistas com os casos e respectivos controles de vizinhança foram realizadas no domicílio de residência. As entrevistas com os controles hospitalares foram realizadas no próprio ambulatório, em sala apropriada e destinada para este fim. Todos os participantes do estudo assinaram um termo de consentimento informado.

Além do sexo e da idade, as variáveis de interesse foram constituídas pelo seguinte elenco: diabetes mellitus; antecedentes familiares de cardiopatia; hipercolesterolemia; hipertensão arterial sistêmica; hábito de fumar; e obesidade. As exposições que expressam "doença" referem-se ao conhecimento prévio da condição a partir do diagnóstico. Os níveis de exposição para as variáveis $h i$ pertensão arterial, hipercolesterolemia e hábito de fumar foram classificados mediante investigação da duração dessas exposições, considerando-se para o hábito de fumar, além da duração, o número de cigarros consumidos/dia $\mathrm{e}$ exposição atual ou pregressa à condição.

A escolha inicial do elenco das possíveis variáveis de confusão foi fundamentada, em princípio, pelo seu reconhecimento em estudos epidemiológicos anteriores, com objetivos similares aos do presente estudo, sendo procu- radas, tanto quanto possível, as bases biológicas que explicassem suas associações com a variável resposta e com as demais variáveis independentes, evitando-se, assim, a inclusão de variáveis intervenientes ${ }^{10}$.

\section{Categorização das Variáveis}

A categoria de referência para a variável sexo foi representada pelos indivíduos do "sexo feminino". A variável idade foi classificada em 4 categorias, com intervalos de 10 anos: "30 a 39"; "40 a 49"; "50 a 59" e "60 a 69 anos". A primeira categoria foi considerada a de referência. As variáveis diabetes mellitus, antecedentes familiares de cardiopatia, hipercolesterolemia e hipertensão arterial sistêmica foram tratadas como dicotômicas ("expostos" e "não expostos"), constituindo-se os "não expostos" as respectivas categorias de referência. $\mathrm{O}$ critério de classificação para a categoria de "expostos" foi baseado no histórico de exposições, e, para o caso da hipertensão arterial, levou-se em consideração o uso atual ou pregresso de medicação antihipertensiva. Para avaliação do efeito dose-resposta, a variável duração da hipertensão arterial foi codificada em dois níveis de exposição: " $\leq 2$ anos" e "> 2 anos" de duração, utilizando-se como ponto de corte a mediana de duração nos controles. A respectiva categoria de referência foi representada pelos "não hipertensos". A duração da hipercolesterolemia foi codificada em dois níveis de exposição: " $\leq 3$ anos" e "> 3 anos" de duração, utilizando-se como ponto de corte a mediana de duração nos controles. A respectiva categoria de referência foi constituída pelos indivíduos "sem história de hipercolesterolemia". O hábito de fumar foi codificado em três categorias: "não fumantes"; "ex-fumantes" e "fumantes", utilizando-se como referência a categoria de "não fumantes". A duração do hábito de fumar foi codificada em dois níveis de exposição: " $\leq 30$ anos" e ">30 anos" de duração, utilizando-se como ponto de corte a mediana de duração nos controles. A categoria de referência foi representada pelos "não fumantes". A variável número de cigarros consumidos/dia foi codificada em dois níveis de exposição: " $\leq 20$ unidades/dia" e "> 20 unidades/dia", utilizando-se como ponto de corte o equivalente a um maço de cigarros. A categoria de referência foi representada pelos "não fumantes".

O critério utilizado para classificação da obesidade foi o índice de massa corporal (peso/altura ${ }^{2}$ ). Para a obtenção das medidas antropométricas foram utilizadas balanças portáteis e antropômetros confeccionados em alumínio, contendo escalas métricas em fibra de vidro. Os indivíduos também foram questionados sobre seu peso 10 anos antes da data da entrevista, servindo esta informação para o cálculo do índice de massa corporal no passado. Os índices de massa corporal (IMC) foram primeiramente classificados em 3 categorias: "normal" (IMC < 25); "sobrepeso" (IMC: 25 a 29,9) e "obeso" (IMC $\geq 30$ ), segundo os critérios recomendados por Bray ${ }^{1}$. A partir desta classificação foi criada a variável $B E V$, codificada em 4 níveis e indicativa da evolução do índice de massa corporal no decorrer dos 10 anos que antecederam a entrevista. Os indivíduos com IMC normal há 10 anos e atualmente 
se constituíram na categoria de referência "nível 1". Os obesos ou com sobrepeso no passado e IMC normal no presente representaram o "nível 2". Os que apresentaram IMC normal no passado e estiveram obesos ou com sobrepeso no presente compuseram o "nível 3". Os indivíduos obesos ou com sobrepeso nos dois momentos representaram o "nível 4" de exposição.

Foram realizadas 900 entrevistas: 300 casos, 300 controles de vizinhança e 300 controles hospitalares. Os registros que contiveram perdas de informações foram excluídos. A amostra remanescente ficou constituída por 833 indivíduos: 280 casos, 285 controles de vizinhança e 268 controles hospitalares. $\mathrm{O}$ poder estatístico foi calculado utilizando-se o modelo proposto por Schlesselman ${ }^{17}$, tendo em vista a detecção de "odds ratios" $\geq 2,0$ em um nível de $5 \%$ monocaudal de significância. $\mathrm{O}$ valor encontrado foi de $95 \%$.

Os dados foram processados em microcomputador na forma de dupla entrada de dados, utilizando-se o programa Foxpro vs. $2.0^{3}$. Utilizou-se programa específico para a análise de consistência interna dos dados.

\section{Análise Estatística}

A técnica estatística utilizada foi a de regressão logística multivariada não condicional ${ }^{8}$ (programa MULTLR $^{2}$ ). Inicialmente foram realizadas análises univariadas, utilizando-se, como critério de exclusão para os modelos multivariados, valores $\mathrm{p}>0,25$ para os testes de Wald. O critério adotado para a seleção de variáveis, a partir dos modelos multivariados, foi a razão de verossimilhança, utilizando-se níveis de significância $\leq 0,05^{6}$. Os testes de tendência para os "odds ratios" de variáveis com mais de duas categorias foram realizados utilizando-se o programa MULTLR².

\section{RESULTADOS}

A distribuição dos casos, segundo sexo e diagnóstico, encontra-se na Tabela 1 . Observa-se que a estrutura por sexo dos casos exibiu uma razão masc./ fem.=2/1. Com relação aos diagnósticos, nota-se um predomínio da angina do peito (código 413) em ambos os sexos. O infarto do miocárdio foi mais freqüente entre os homens. Com relação aos outros diagnósticos, observa-se uma distribuição similar para ambos os sexos.
Tabela 1 - Número e percentagem de casos, segundo sexo e diagnóstico.

\begin{tabular}{|c|c|c|c|c|}
\hline \multicolumn{5}{|c|}{ Sexo } \\
\hline & Diagnóstico & Masculino & Feminino & Total \\
\hline & $(\mathrm{CID})^{*}$ & $\mathrm{~N}^{\circ}(\%)$ & $N^{\circ}(\%)$ & $\mathrm{N}^{\circ}(\%)$ \\
\hline & 410 & $33(17)$ & $6(7)$ & $39(14)$ \\
\hline & 412 & $5 \quad(3)$ & $2 \quad(2)$ & $7 \quad(3)$ \\
\hline & 413 & $113(59)$ & $64(73)$ & $177(63)$ \\
\hline & 414 & $41(21)$ & $16(18)$ & $57(20)$ \\
\hline & Total & $192(100)$ & $88(100)$ & $280(100)$ \\
\hline $\begin{array}{l}* \text { CID } \\
410 \\
412 \\
413 \\
414\end{array}$ & $\begin{array}{l}\text { Classificação I } \\
\text { Infarto agudo } \\
\text { Infarto antigo } \\
\text { Angina do pei } \\
\text { Outras formas }\end{array}$ & $\begin{array}{l}\text { cional de Doenç } \\
\text { cádio } \\
\text { cárdio }\end{array}$ & s- IX Revisão & \\
\hline
\end{tabular}

A Tabela 2 refere-se à distribuição de casos e controles segundo sexo e faixa etária. Nota-se um razoável equilíbrio nas proporções de casos e respectivos controles segundo estas variáveis demográficas.

A Tabela 3 refere-se à distribuição de casos e controles segundo as variáveis de exposição.

A análise dos dados foi procedida em três etapas: a) análises univariadas; b) modelos multivariados contendo as categorias de duração das variáveis de interesse; c) modelos finais.

As variáveis sexo e faixa etária não foram cogitadas na análise em virtude de se ter procedido ao emparelhamento de casos e controles, para estas variáveis, na fase de delineamento do estudo.A variável $B E V$ (evolução do índice de massa corporal) foi excluída dos modelos finais por não apresentar significância estatística.

Nas Tabelas 4, 5, 6 e 7 estão apresentados os odds ratios brutos e ajustados, com os respectivos intervalos de confiança, para as variáveis que compuseram os modelos finais. Nota-se que cada modelo final foi composto, respectivamente, pelos níveis de exposição das variáveis duração do hábito de fumar, número de cigarros consumidos/dia, duração da hipercolesterolemia e duração da hipertensão arterial, ajustados para os potenciais fatores de confusão. Observa-se que os odds ratios das variáveis número de cigarros consumidos/dia e duração da hipertensão arterial apresentaram um gradiente linear, segundo os níveis de exposição. O mesmo não se ob-

Tabela 2 - Número e percentagem de casos e controles, segundo sexo e faixa etária.

\begin{tabular}{|c|c|c|c|c|c|}
\hline \multirow[b]{2}{*}{ F. Etária } & \multicolumn{2}{|c|}{ Masculino } & \multicolumn{2}{|c|}{ Feminino } & \multirow[b]{2}{*}{$\begin{array}{c}\text { Total } \\
\text { No }^{\circ}(\%)\end{array}$} \\
\hline & $\begin{array}{c}\text { Caso } \\
\mathrm{N}^{\mathrm{O}}(\%) \\
\end{array}$ & $\begin{array}{c}\text { Controle } \\
N^{\circ}(\%)\end{array}$ & $\begin{array}{c}\text { Caso } \\
N^{\circ}(\%)\end{array}$ & $\begin{array}{c}\text { Controle } \\
N^{\circ}(\%)\end{array}$ & \\
\hline 30-39 anos & $31(16,1)$ & $58(16,3)$ & $7(8,0)$ & $20(10,1)$ & $116(13,9)$ \\
\hline 40-49 anos & $53(27,6)$ & $93(26,2)$ & $26(29,5)$ & $61(30,8)$ & $233(28,0)$ \\
\hline 50-59 anos & $99(51,6)$ & $184(51,8)$ & $46(52,3)$ & $97(49,0)$ & $426(51,1)$ \\
\hline 60-69 anos & $9(4,7)$ & $20(5,6)$ & $9(10,2)$ & $20(10,1)$ & $58(7,0)$ \\
\hline Total & $192(100)$ & $355(100)$ & $88(100)$ & $198(100)$ & $833(100)$ \\
\hline
\end{tabular}


Tabela 3 - Número e porcentagem de casos e controles segundo variáveis de exposição.

\begin{tabular}{|c|c|c|}
\hline Variável & $\begin{array}{c}\text { Caso } \\
N^{\circ}(\%)\end{array}$ & $\begin{array}{c}\text { Controle } \\
\mathrm{N}^{\circ}(\%)\end{array}$ \\
\hline \multicolumn{3}{|l|}{ Diabetes } \\
\hline Sem história* & $232(82,9)$ & $509(92,0)$ \\
\hline Com história & $48 \quad(17,1)$ & $44(8,0)$ \\
\hline \multicolumn{3}{|l|}{ AFC } \\
\hline Com história & $204(72,9)$ & $260(47,0)$ \\
\hline \multicolumn{3}{|l|}{ Háb. fumar } \\
\hline Não fumante* & $82(29,3)$ & $236(42,7)$ \\
\hline Ex-fumante & $121(42,3)$ & $153(27,7)$ \\
\hline Fumante & $77(27,5)$ & $164(29,7)$ \\
\hline \multicolumn{3}{|l|}{ Duração fumo } \\
\hline Não fumante* & $82 \quad(29,3)$ & $236(42,7)$ \\
\hline$\leq 30$ anos & $125(44,6)$ & $198(35,8)$ \\
\hline$>30$ anos & $73(26,1)$ & $119(21,5)$ \\
\hline \multicolumn{3}{|l|}{$\mathrm{N}^{\circ}$ cigarros/dia } \\
\hline Não fumante $(*)$ & $82(29,3)$ & $236(42,7)$ \\
\hline$\leq 20$ unidades & $127(45,4)$ & $246(44,5)$ \\
\hline$>20$ unidades & $71(25,4)$ & $71(12,8)$ \\
\hline \multicolumn{3}{|l|}{ BEV } \\
\hline Normal/normal* & $61(21,8)$ & $194(35,1)$ \\
\hline Obeso/normal & $17 \quad(6,1)$ & $27 \quad(4,9)$ \\
\hline Normal/obeso & $86(30,7)$ & $145(26,2)$ \\
\hline Obeso /obeso & $116(41,4)$ & $187(33,8)$ \\
\hline \multicolumn{3}{|l|}{ H. hipercol. } \\
\hline Sem história* & $165(58,9)$ & $470(85,0)$ \\
\hline Com história & $115(41,1)$ & $83(15,0)$ \\
\hline \multicolumn{3}{|l|}{ Duração hipercol. } \\
\hline Sem história* & $165(58,9)$ & $470(85,0)$ \\
\hline$\leq 3$ anos & $62(22,1)$ & $49(8,9)$ \\
\hline$>3$ anos & $53(18,9)$ & $34 \quad(6,1)$ \\
\hline Sem história* & $91(32,5)$ & $388(70,2)$ \\
\hline Com história & $189(67,5)$ & $165(29,8)$ \\
\hline \multicolumn{3}{|l|}{ Duração HAS } \\
\hline Sem história* & $91(32,5)$ & $388(70,2)$ \\
\hline$\leq 2$ anos & $83(29,6)$ & $91(16,5)$ \\
\hline$>2$ anos & $106(37,9)$ & $74(13,4)$ \\
\hline categoria de ref & & \\
\hline AFC & de cardiopatia & \\
\hline índice de Bray em & asiões: há 10 anos & atualmente \\
\hline história de hiperte & arterial sistêmica & \\
\hline história de hipercc & olemia & \\
\hline
\end{tabular}

servou para os odds ratios das variáveis duração do hábito de fumar e duração da hipercolesterolemia. Os odds ratios para a variável diabetes (histórico de exposição), embora significantes nos modelos univariados, perderam magnitude e significância estatística após o ajustamento para as demais variáveis contidas nos modelos finais. Os odds ratios das variáveis hábito de fumar, antecedentes familiares de cardiopatia, história de hipertensão arterial e história de hipercolesterolemia mantiveram significância estatística mesmo após o ajustamento para os potenciais fatores de confusão.

\section{DISCUSSÃO}

De acordo com os resultados apresentados nas Tabelas 4 e 6 (modelos multivariados) os odds ratios para as categorias de exposição das variáveis duração do hábito de fumar e duração da hipercolesterolemia, embora significantes, não foram indicativos de um gradiente linear. A distribuição dos indivíduos nas categorias de duração do hábito de fumar incluiu "ex-fumantes" e "fumantes" que foram agrupados segundo a duração do hábito. É possível que tal agrupamento tenha contribuído para o achado de medidas de efeito similares, visto que o hábito de fumar, quando tratado como tal ("não fumantes", "ex-fumantes" e "fumantes"), exibiu odds ratios de maior magnitude para a categoria de "ex-fumantes" comparados aos "não fumantes", do que para a de "fumantes" quando comparados aos "não fumantes" (Tabelas 6 e 7). O achado de odds ratios de maior magnitude para a categoria de

Tabela 4 - Odds Ratios brutos e ajustados com respectivos intervalos de confiança (95\%), segundo as variáveis do estudo e o tipo de análise.

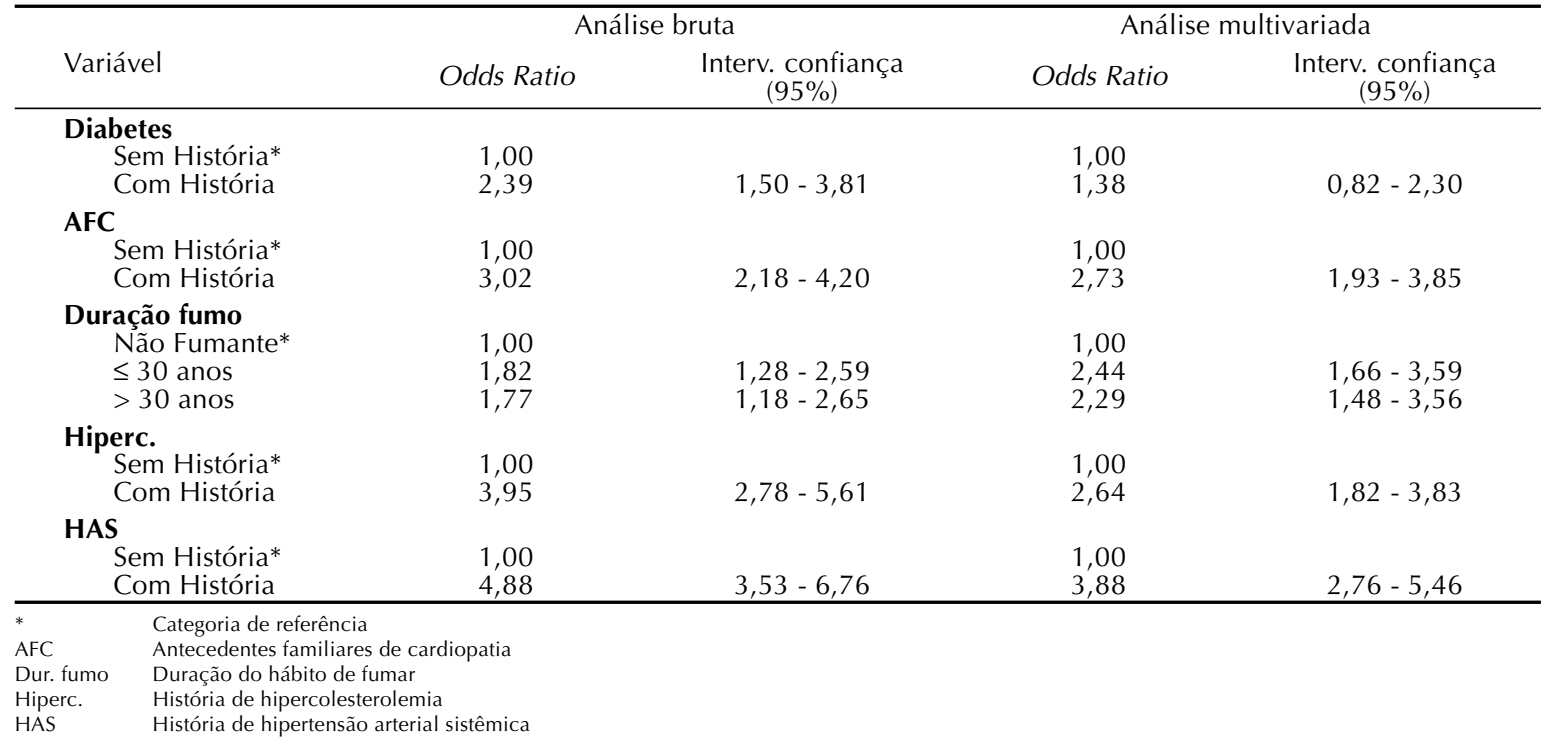


Tabela 5 - Odds Ratios brutos e ajustados com respectivos intervalos de confiança (95\%) segundo as variáveis do estudo e o tipo de análise.

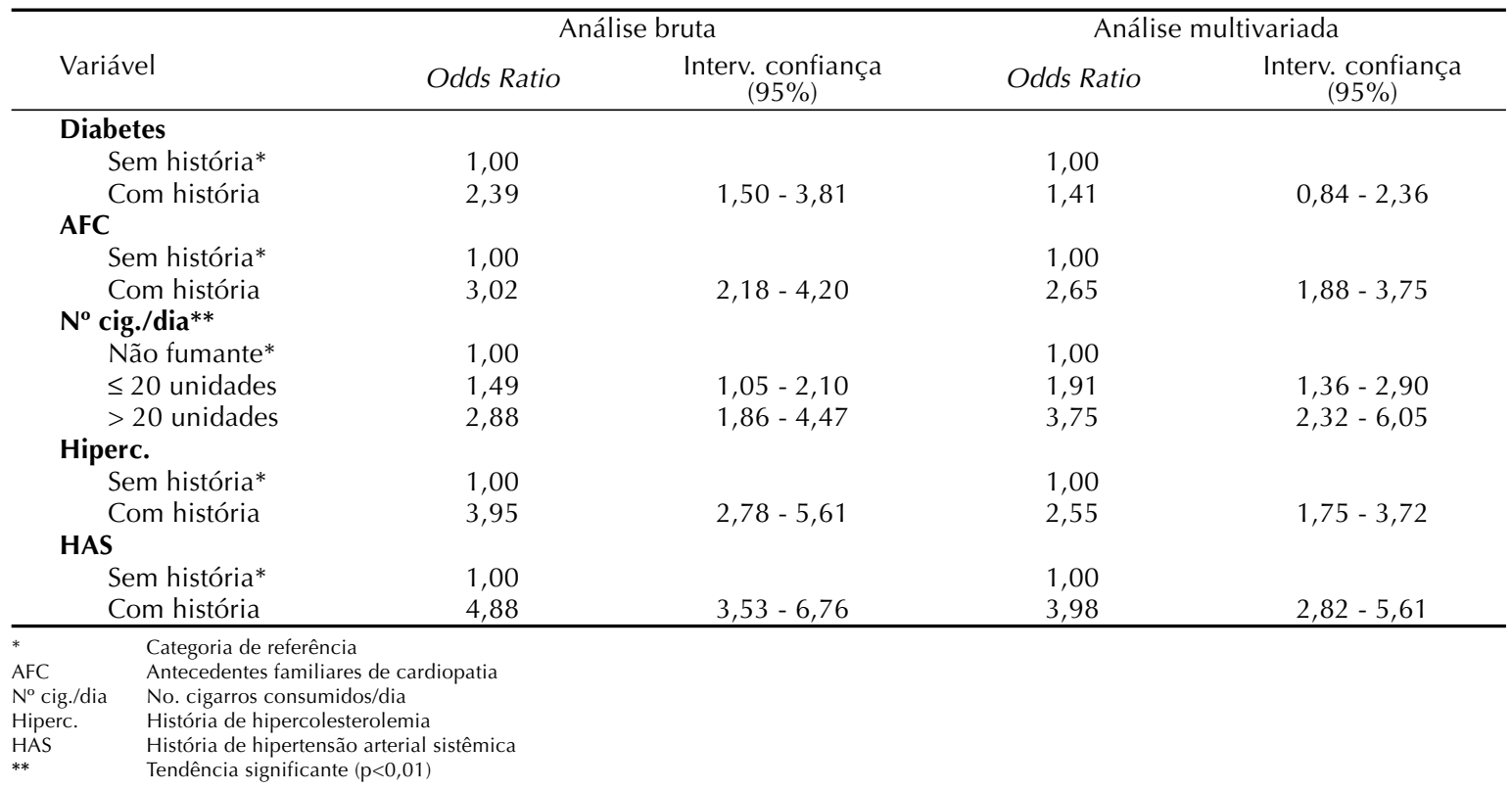

Tabela 6 - Odds Ratios brutos e ajustados com respectivos intervalos de confiança (95\%) segundo as variáveis do estudo e o tipo de análise.

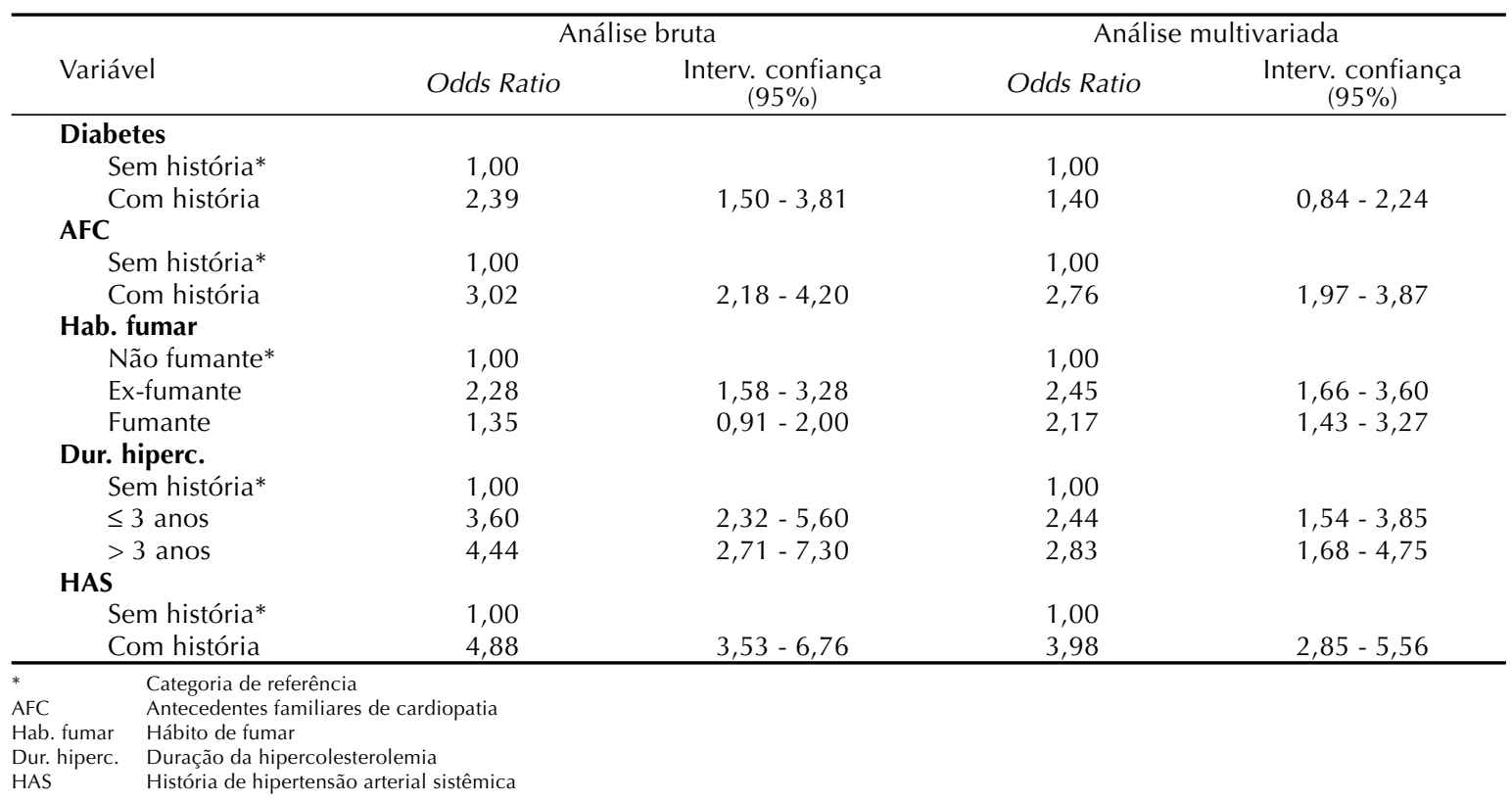

ex-fumantes, por outro lado, pode ser explicado pela inclusão de casos prevalentes no estudo, com possíveis modificações no hábito de fumar a partir de um diagnóstico prévio de DIC.

Com relação à duração da hipercolesterolemia é possível ter ocorrido algum viés de memória entre os respondentes, por se tratar de condição por vezes assintomática, e só passível de detecção através de exame laboratorial. Não obstante, os odds ratios para as categorias de duração desta variável apresentaram significância estatística, embora, sem indicação de um gradiente linear. A comparação com outros estudos ficou prejudicada por não se ter encontrado, em publicações dos últimos dez anos, trabalhos que relacionassem o efeito da duração da hipercolesterolemia com a DIC. 
Tabela 7 - Odds Ratios brutos e ajustados com respectivos intervalos de confiança (95\%) segundo as variáveis do estudo e o tipo de análise.

\begin{tabular}{|c|c|c|c|c|c|}
\hline & & & ruta & Anál & variada \\
\hline Vari & & Odds Ratio & $\begin{array}{c}\text { Interv. confiança } \\
(95 \%)\end{array}$ & Odds Ratio & $\begin{array}{c}\text { Interv. confiança } \\
(95 \%)\end{array}$ \\
\hline Dia & tes & & & & \\
\hline & em história* & 1,00 & & 1,00 & \\
\hline & om história & 2,39 & $1,50-3,81$ & 1,30 & $0,77-2,20$ \\
\hline AFC & & & & & \\
\hline & em história* & 1,00 & & 1,00 & \\
\hline & om história & 3,02 & $2,18-4,20$ & 2,72 & $1,92-3,84$ \\
\hline $\mathrm{Hab}$ & fumar & & & & \\
\hline & ão fumante* & 1,00 & & 1,00 & \\
\hline & «-fumante & 2,28 & $1,58-3,28$ & 2,71 & $1,82-4,03$ \\
\hline & umante & 1,35 & $0,91-2,00$ & 2,07 & $1,36-3,15$ \\
\hline Hip & & & & & \\
\hline & história* & 1,00 & & 1,00 & \\
\hline & om história & 3,95 & $2,78-5,61$ & 2,54 & $1,75-3,71$ \\
\hline Dur & HAS** & & & & \\
\hline & em história* & 1,00 & & 1,00 & \\
\hline & 2 anos & 3,89 & $2,62-5,78$ & 3,32 & $2,21-4,97$ \\
\hline & 2 anos & 6,11 & $4,11-9,08$ & 4,44 & $2,92-6,75$ \\
\hline * & Categoria de re & & & & \\
\hline & Antecedentes fa & ardiopatia & & & \\
\hline Hab. fuma & Hábito de fuma & & & & \\
\hline Hiperc. & História de hip & & & & \\
\hline Dur. HAS & Duração da hip & ial sistêmica & & & \\
\hline ** & Tendência sign & & & & \\
\hline
\end{tabular}

As Tabelas 5 e 7 indicaram, respectivamente, que tanto o número de cigarros consumidos/dia como a duração da hipertensão arterial apresentaram um efeito dose-resposta na produção da doença isquêmica do coração. Tais efeitos também foram relatados por outros autores $\left(\mathrm{He}^{5}\right.$, Nayak ${ }^{13}$, Ramsdale ${ }^{16}$, Puschmayer ${ }^{15}$ e Stevanov $\left.{ }^{19}\right)$.

Ao se proceder, na fase de delineamento do estudo, ao emparelhamento, por faixa etária, de casos e controles, procurou-se minimizar o efeito do envelhecimento na determinação do risco de doença, de sorte que os odds ratios calculados pudessem refletir o efeito de diferentes níveis destas exposições sobre a DIC, independentemente da idade. Destacase também que a indicação de gradação para as variáveis número de cigarros consumidos/dia e duração da hipertensão arterial persistiram mesmo após o ajustamento para as variáveis de confusão.

Os casos elegíveis para o estudo foram selecionados por ordem cronológica de alta hospitalar, até que se completasse o número previsto para o tamanho da amostra. Os indivíduos elegíveis que não participaram do estudo em decorrência de óbito, recusa ou não localização foram substituídos, e, para esta substituição, respeitou-se a "ordem natural" de acesso ao hospital estudado, o que, de per si, dificultaria a introdução de vícios de seleção de casos; as substituições não foram procedidas considerando-se, a priori, a disponibilidade, por parte destes casos, em participar do estudo, e sim, o pré- requesito da ordem cronológica de alta hospitalar. Desta forma, o "ambiente" de onde foram selecionados os "casos substitutos" oferecia, teoricamente, oportunidade de participação de indivíduos comparáveis aos excluídos.

Por outro lado, acredita-se que os indivíduos que foram a óbito $(n=16)$, embora considerados casos mais graves, pouco teriam contribuído para o estudo, visto representarem uma perda de $5 \%$ e, mesmo assim, com probabilidade de uma substituição equivalente em relação ao prognóstico.

Os resultados do estudo confirmam, pois, a relevância da hipertensão arterial, da hipercolesterolemia e do hábito de fumar como fatores de risco para a doença isquêmica do coração, além de indicarem o efeito deletério do tempo de exposição à hipertensão arterial e da quantidade de cigarros consumidos por dia.

\section{AGRADECIMENTOS}

Ao Prof. Dr. José Antonio Franchini Ramires, Diretor da Divisão de Coronariopatias de INCORFMUSP, pelo empenho quanto ao acesso à instituição para a seleção dos casos do estudo; ao Dr. Paulo Andrade Lotufo, Diretor do Ambulatório Geral e Didático do Hospital das Clínicas-HC-FMUSP, por facilitar o acesso a esse Ambulatório para a seleção dos controles hospitalares; aos relatores, pelas sugestões e comentários que muito contribuíram para a versão final do presente trabalho. 


\section{REFERÊNCIAS BIBLIOGRÁFICAS}

1. BRAY, G. A. Obesity: basic considerations and clinical approaches. Dis. Month, 35 (7): 451-537, 1989.

2. CAMPOS-FILHO, N. \& FRANCO, E. L. MULTLR-A microcomputer program for multiple logistic regression by unconditional and conditional maximum likelihood methods. Am. J. Epidemiol., 129: 439-44. 1989.

3. FOX HOLDINGS INC. FOXPRO vs. 2.0. C./S. Pat. Pend American Edition, 1991.

4. GOLDSTEIN, M. R. Cholesterol inibition, cancer and coronary heart disease. Lancet, 340: 127-8, 1992.

5. HE, Y. Hypertension and angiographically defined coronary heart disease. Chung. Hua. Liu. Hsing. Ping. Hsueh. Tsa. Chih., 10: 282-6,1989.

6. HOSMER, D. W. \& LEMESHOW, S. Applied logistic regression. New York, John Wiley \& Sons, 1989.

7. KANNEL, W. B. Hypertension as a risk factor for cardiac events. Epidemiologic results of long-term studies. $J$. Cardiovasc. Pharmacol., 21: S27- S37 - 1993.

8. KLAINBAUM, D. G. et al. Applied regression analysis and other multivariable methods. Boston, PWS-KENT Publishing Company, 1988.

9. LOLIO, C. A. et al. Decline in cardiovascular disease mortality in the city of São Paulo, Brazil, 1970 to 1983. Rev. Saúde Pública, 20: 454-64, 1986.

10. MIETTINEN, O. S. \& COOK, E. F. Confouding: essence and detection. Am. J. Epidemiol., 114: 593-603, 1981.

11. MORAES, S. A. \& SOUZA, J. M. P. Diabetes mellitus e doença isquêmica do coração: comparação por sexo. Arq. Bras. Cardiol., 66: 59-63, 1996.
12. MORAES, S. A. \& SOUZA, J. M. P. Diabetes mellitus e doença isquêmica do coração. Um estudo tipo caso-controle. Rev. Saúde Pública, 30: 364-71, 1996.

13. NAYAK, K.C. et al. Treadmill exercise testing in asymptomatic chronic smokers to detect latent coronary heart disease. Indian Heart J., 41: 62-5, 1989.

14. ORGANIZAÇÃO MUNDIAL DA SAÚDE. Manual da classificação estatística internacional de doenças, lesões e causas de óbito. São Paulo, Centro da O.M.S. para Classificação ds Doenças em Português, 1980.

15. PUSHMAYER, V. Smoking as a risk factor for the development of arterial occlusive disease. Acta. Univ. Carol., [Med.] (Praha), 105: 1-134, 1984.

16. RAMSDALE, D. R. et al. Smoking and coronary heart disease assessed by routine coronary arteriography. Br. Med. J., 290: 197-200, 1985

17. SCHLESSELMAN, J. J. Case control studies-design, conduct and analysis. New York, Oxford University Press, 1982.

18. SINNET, P. \& WHITE, M. Lifestyle health and disease: a comparison between Papua New Guinea and Australia. Med. J. Aust., 1: 1-5, 1978.

19. STEVANOV, G. et al. Hypertension incidence and its reflection in vascular and organic lesions in 9014 autopsies of subjects over 14 years of age. Vutr. Boles., 20: 54-60, 1981.

20. THE EPIDEMIOLOGICAL transition. [Editorial] Lancet, 2: $670,1977$.

21. THERRIEN J. M. \& LAVIE, C. J. Smoking and ischaemic heart disease. Postgrad. Med., 91: 74-6, 1992. 